\title{
Refilling of Geosynchronous Flux Tubes as Observed at the Equator by GEOS 2
}

\author{
J. J. SOJKA' AND G. L. WRENN
}

Mullard Space Science Laboratory, University College London, Holmbury St. Mary, Dorking, Surrey, England

\begin{abstract}
During periods of extended quiet geomagnetic activity the geosynchronous satellite orbit lies inside the plasmasphere. Five such periods were observed by the GEOS 2 satellite. During the initial 48 hours of such periods the equatorial plasma flux tube density increases at 30 to $50 \mathrm{~cm}^{-3} /$ day. However, on reaching $\sim 100$ $\mathrm{cm}^{-3}$ the refilling rate decreases, and refilling is limited. Only when the density reaches $\sim 100 \mathrm{~cm}^{-3} \mathrm{do}$ the plasma characteristics and fluctuations appear to be plasmaspheric and the flow predominantly corotational. The "hot outer zone" of the plasmasphere is highly structured in density and temperature when viewed from a corotating satellite. This region also has a relatively dense population of warm subkilovolt electrons. These warm electrons whose density is $\sim 1 \%$ to $50 \%$ of the cold plasma may be the heat source for the hot outer zone ions.
\end{abstract}

\section{INTRODUCTION}

Refilling of the plasmasphere occurs after plasmaspheric flux tubes have been depleted by magnetospheric storms. These plasma flux tubes refilled over a period of several days depending upon the severity of the storm. The general characteristics of the refilling rate have been inferred from whistler observations [Carpenter and Park, 1973; Park, 1974] and OGO 5 plasma measurements [Chappell, 1972]. This refilling process is caused by plasma from the ionosphere expanding along magnetic flux tubes into the depleted plasma trough; in-situ measurements have been made of the characteristics of the upstreaming thermal ions which are responsible for the refilling process [Sojka et al., 1983; Wrenn et al., 1983].

Theoretical models of both a kinetic and hydrodynamic nature have been used to study the expansion of ionospheric plasma to higher altitudes along magnetic flux tubes [Banks and Holzer, 1969; Marubashi, 1970; Holzer et al., 1971; Lemaire and Scherer, 1974; Raitt et al., 1975, 1977; Schunk and Watkins, 1982]. These models in general allow for both subsonic and supersonic outflow velocities for the ionospheric plasma as it enters the collisionless regions above 2000 to 3000 $\mathrm{km}$. The particular character of the outflow is very sensitive to the boundary conditions applied to the model [Schunk and Watkins, 1982]. Unfortunately, very limited data are available concerning these boundary conditions. In the lower altitude collisional regions the light ion outflow fluxes have been observed [Hoffman and Dodson, 1980]; however, these observations are not able to uniquely define the outflow characteristics. Observations of anisotropic warm (a few to tens of electron volts) ions have been made in the outer plasmasphere [Bezrukikh and Gringauz, 1976; Horwitz and Chappell, 1979; Horwitz et al., 1981; Chappell, 1982; Chappell et al., 1982; Sojka et al., 1983; Wrenn et al., 1983]. Such warm plasmas and their associated pitch angle distributions indicate the presence of significant ion heating. At present these observations, in particular the heat source, cannot be explained with the existing ionospheric outflow models.

'Permanent1

Utah Stanently at Center for Atmospheric and Space Sciences, tah State University, Logan.

Copyright 1985 by the American Geophysical Union.

Paper number 4A8307.

$0148-0227 / 85 / 004 \mathrm{~A}-8307 \$ 05.00$
In this paper we study the refilling characteristics and the plasmapause environment by in-situ observations at the geosynchronous orbit. The observations were made by the suprathermal plasma analyzers (SPA) on the geosynchronous GEOS 2 satellite. Over the initial three years, this satellite at $6.6 R_{E}$ had experienced about five extended magnetically quiet periods each of more than 2 days. During these periods the geosynchronous plasma flux tubes refill and become part of the plasmasphere. On entering the plasmasphere it is expected that their motion will become more corotational as the magnetospheric $\mathbf{E}$ field becomes shielded near the plasmapause environment. Since GEOS 2 is corotating, it provides a unique opportunity to investigate this transport property of the refilling plasma flux tubes. In addition, as the satellite is continually situated in the outer plasmasphere during the quiet periods, a search can be made for the outer plasmasphere ion heat source.

\section{THE SPA INSTRUMENT}

The suprathermal plasma analyzers (SPA) flown on the GEOS 2 satellite are described in detail by Wrenn et al. [1981]. Further background to the operations, analyzer calibration, and in-orbit performance can be found in references contained therein. Two hemispherical electrostatic analyzers are housed in a sensor package which is mounted on a radial boom at a distance of $1.7 \mathrm{~m}$ from the cylindrical satellite body $\left(R_{\mathrm{o}}=0.8 \mathrm{~m}\right)$. Each hemispheric analyzer has an $11 \%$ energy resolution and covers the electron and proton energy range 0.5 to $500 \mathrm{eV}$. Analyzer $\mathrm{A}$ has an $18^{\circ} \times 18^{\circ}$ aperture which views antiparallel to the satellite spin axis, and detector $\mathrm{B}$ has a $32^{\circ} \times 8^{\circ}$ aperture viewing at $100^{\circ}$ to the spin axis. The analyzers have no mass discrimination. It is assumed that protons are the major species.

\section{OBSERVATIONS}

For most of the GEOS 2 operational life, in excess of three years, the SPA instrument collected data from a standard survey mode once every $15 \mathrm{~min}$. Because of the geosynchronous nature of the orbit these data were collected at all local times every day. From these data, several basic plasma parameters characterizing different populations can be monitored at $15 \mathrm{~min}$ resolution over many days. The aim of this study is to observe the characteristics of the plasmasphere at the geosynchronous location of GEOS 2 and study its "corotational" properties. 
GEOS 2 SPA

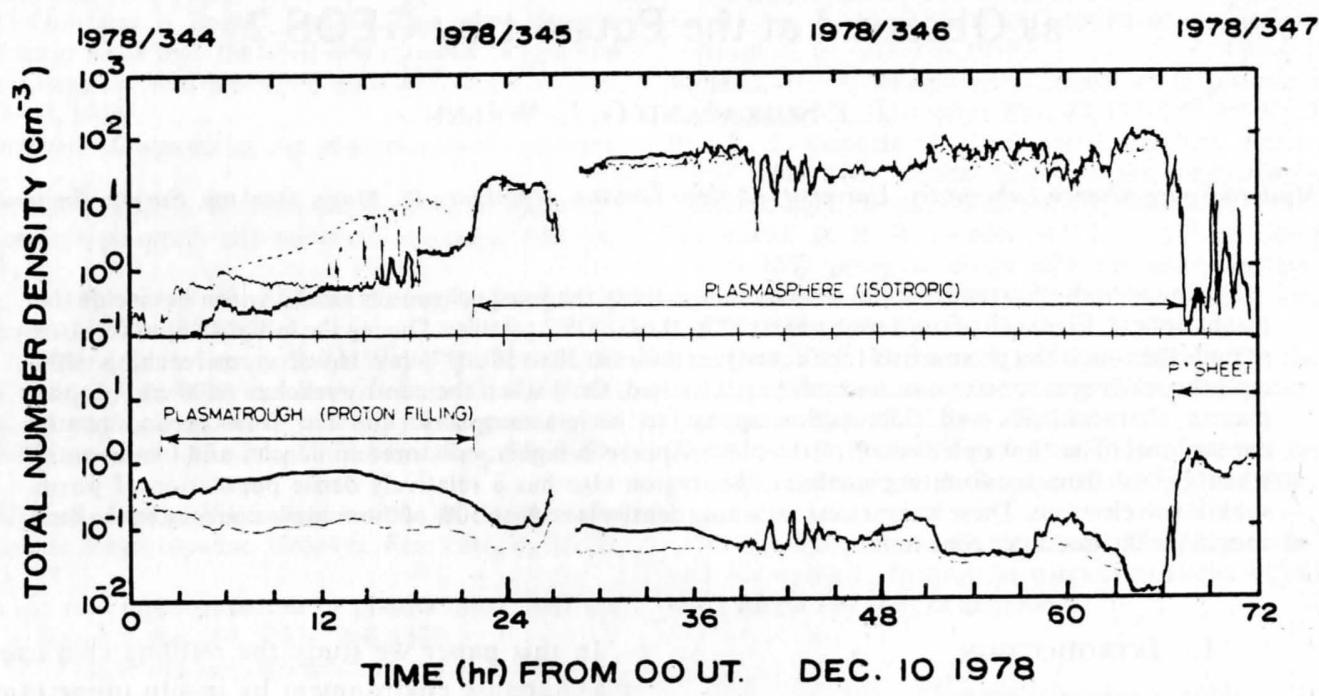

Fig. 1. Thermal ion (top panel) and 50 to $509-\mathrm{eV}$ electron (bottom panel) densities over a 3-day period beginning at $0000 \mathrm{UT}$ on December 10,1978 . The light line represents detector $A$ data (pitch angle range $12^{\circ}$ to $20^{\circ}$ ) while the heavy line represents detector B data covering $60^{\circ}$ to $110^{\circ}$ pitch angles.

Hence the cold plasmaspheric plasma and the adjacent warmer plasma trough-inner edge of the plasma sheet plasma parameters will be presented.

In the plasmasphere the temperature of the cold plasma is on the order of 0.5 to $1 \mathrm{eV}$, and the satellite potential is about one volt negative. At these low temperatures and retarding sheath potentials it is very difficult on a routine basis to extract information on the electron plasma component; in contrast the SPA readily observes the thermal ion component. To observe the cold ions, a negative bias voltage is applied to the boommounted SPA package to locally overcome the effects of any retarding sheath potential [Sojka et al., 1984]. This bias voltage ranges from $-2 \mathrm{~V}$ to $-28 \mathrm{~V}$ from the dense plasmasphere to the plasma trough. The magnitude of the bias voltage is determined by a real time program designed to optimize the statistical significance of the count rate (by increasing bias) and maintain good energy sensitivity (by decreasing bias). The thermal ion analysis is able to yield not only density but also temperature, satellite potential, and angular anisotropy [Wrenn et al., 1983]. The ion densities and temperature derived from the SPA are consistent with those observed by other experiments on GEOS 2 [Decreau et al., 1978]. In the plasmapause region in addition to a thermal plasma component there is also a warmer component of relatively low density with energy in the tens of $\mathrm{eV}$ to $100 \mathrm{eV}$ range. This population is also used to identify the inner edge of the plasma sheet [Frank, 1971]. Since this plasma population is warm but has a low density, the SPA in the ion mode is quite insensitive to it. However, in the electron mode, significant fluxes are found over the whole instrument energy range 0.5 to $500 \mathrm{eV}$. For energies below about $50 \mathrm{eV}$ the count rates are enhanced by local satellite sheath electrons and, on occasion, escaping ionospheric photoelectrons. To avoid the difficult task of deconvoluting these other $<50-\mathrm{eV}$ electron sources, the SPA survey mode for warm plasmapause electrons ranged from 50 to $500 \mathrm{eV}$. Over this energy range the plasma distribution function was integrated to give an electron density. This integration was performed separately for each detector, and by assuming pitch angle isotrophy in each case a density was obtained. Since the detectors view approximately parallel and perpendicular to the magnetic field line, these two densities give an indication of the actual plasma isotropy.

In Figure 1 the cold ion density (top panel) and warm electron density (bottom panel) are shown for a 3-day period beginning at 0000 UT on December 10, 1978. The heavy solid line represents the density inferred from analyzer $B$, which views $60^{\circ}$ to $110^{\circ}$ pitch angles, while the light solid line is the densities inferred from analyzer A viewing small pitch angles. For the cold ion density the isotropic distribution function was integrated from 0 to $\sim 8 \mathrm{eV}$ while for the warm electron density the integral is from 50 to $500 \mathrm{eV}$. Initially at $0000 \mathrm{UT}$ until about 1200 UT the cold ion density and warm electron density are comparable, ranging from 0.1 to $1 \mathrm{~cm}^{-3}$. Their angular properties are quite different. The cold ions are field aligned by a factor of 2 to 8 while the warm electrons show a "pancake" distribution with the perpendicular density exceeding the parallel by a factor of 2 to 10 . These two populations are characteristic of the plasma trough [see Wrenn et al., 1979; Sojka et al., 1984]. During this time the plasmapause is earthward of the GEOS 2 geosynchronous location. The local time in Figure 1 is given by UT +2 hours, $24 \mathrm{~min}$; hence this first 12-hour period corresponds to 0224 to $1424 \mathrm{LT}$. Between 0600 and $1500 \mathrm{UT}$ the field-aligned (light line) cold proton density is augmented by a dashed line. When the satellite is in the plasma trough, the spacecraft potential becomes positive up to about 5 $\mathrm{V}$ and causes the field-aligned detector to show marked spin modulation. As a consequence the density integration yields a density which is severely reduced. A full discussion of this effect is given by Sojka et al. [1984]. The dashed line represents a more probably variation for the field-aligned density during this period.

From 1200 to 2200 UT in Figure 1 the cold ion density, although still field aligned, increases to about $10 \mathrm{~cm}^{-3}$. During this time the warm electron density decreases to about $0.1 \mathrm{~cm}^{-3}$. Also, the cold plasma density appears to be increasing toward plasmapause values, $\approx 10 \mathrm{~cm}^{-3}$. Because plasma flux tubes do not corotate in the plasma trough, a region with a significant convection electric field, GEOS 2 is not following a single plasma flux tube. Hence this apparent refilling cannot be 


\section{GEOS-2 SPA}

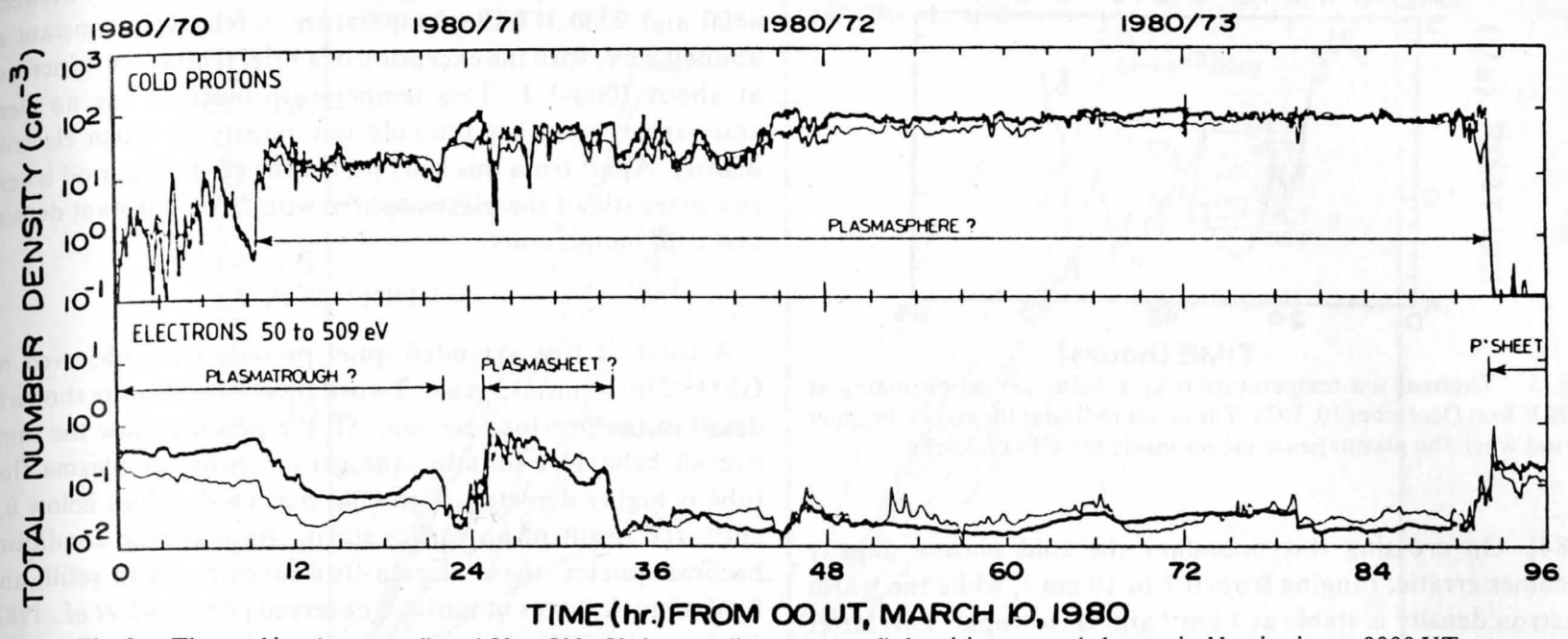

Fig. 2. Thermal ion (top panel) and 50 to $509-\mathrm{eV}$ electron (bottom panel) densities over a 4-day period beginning at $0000 \mathrm{UT}$ on March 10, 1980. The light line represents detector A data (pitch angle range $11^{\circ}$ to $28^{\circ}$ ) while the heavy line represents detect or B data covering $50^{\circ}$ to $120^{\circ}$.

related to the refilling of a single plasma flux tube. These data do, however, reflect the prevailing very quiet magnetospheric conditions; from 0000 UT until 6000 UT the 3-hourly Kp remained 0 or $0+$. Over this very quiet two and a half day period the plasmasphere was refilling, and the plasmapause moved out beyond GEOS 2.

At 2200 UT the cold plasma density had reached $\sim 20 \mathrm{~cm}^{-3}$ and had become isotropic. This density reasonably identifies a cold plasma plasmapause; however, no equivalent signature is present in the warm electron density. From 2400 to 6000 UT, GEOS 2 is inside the "plasmasphere." Inside the plasmasphere the flux tube motion is dominated by corotation, although the magnetospheric electric field can still introduce departures from a pure corotation motion. Since GEOS 2 is corotating, it can be used as a reference point about which density fluctuations can be studied to see if any sensible departure from corotation can be identified. If the plasma were primarily corotating, the plasma would be isotropic, and if refilling were present, the density would increase with time. At 2400 UT the density is $\sim 20 \mathrm{~cm}^{-3}$, while at $6600 \mathrm{UT}$ it has reached 80 to 110 $\mathrm{cm}^{-3}$, and the plasmasphere has undergone refilling. Unfortunately, the interim period is filled with large scale density fluctuations as well as cold plasma anisotropies. The cold plasma shows a marked tendency to have larger perpendicular than parallel densities, i.e., between 2400 and 4000 UT and again between 5000 and 6600 UT in Figure 1. At other times the cold plasma appears isotropic, i.e., between 4000 and 5000 UT in Figure 1 and between 7500 and 8700 UT in Figure 2. Since the perpendicular to parallel density differences are typically a $20 \%$ effect, it has not been possible to uniquely identify the source of the anisotropy. This magnitude is somewhat larger than expected from the relative instrumental intercalibration uncertainty, especially since the anisotropy varies in a way more coupled to other geophysical fluctuations. Intercalibrating two detectors where one is almost always looking parallel to the magnetic field while the other is nearly perpendicular is difficult. However, the fluctuation observed and especially their variation from hour to hour are strongly indicative of the presence of some external plasma anisotropy. These aniso- tropies could arise from either a pitch angle anisotropy or a drift in the equatorial plane. Either mechanism indicates a nonclassical plasmasphere behavior, and the latter (a drift in the equatorial plane) would indicate the presence of a convection electric field inside the plasmapause. In addition to this nonisotropic nature the plasma density also fluctuates markedly. Over relatively short periods ( $15 \mathrm{~min}$ resolution) the density changes by a factor of 10 (see Figure 1, top panel, at $\sim 4100$ UT). Indeed, the density can oscillate relatively systematically; between 4000 and 4800 UT a total of six well-defined dips are present. At other times the density fluctuates by up to a factor of 2 about a mean trend value. This trend value for the 2400 to 6000 UT period does not monotonically increase; it shows periods of decreasing density.

During the period when the cold ion density is about $100 \mathrm{~cm}^{-3}$ the warm electron density is low, $\sim 0.1 \mathrm{~cm}^{-3}$; however, it shows marked modulation. Indeed, these modulations appear to be inversely related to variations in the cold ion density. In the 4000 to 4800 UT period when the cold ions showed systematic "oscillatory" variations the warm electrons also showed such oscillations. The warm electrons originate in the plasma sheet from which they have primarily ExB drifted earthward in the night sector and then away from the earth on the dayside. Local noon occurs at 3824 and 6224 UT during this period. About neither period is there a marked decrease in the warm electron density after midday. Their sustained presence over 36 hours would indicate that they have become trapped in the plasmasphere. This implies that the convection electric field is now too small for them to significantly ExB drift back into the magnetosphere on the dayside.

Over the remaining 12 hours in Figure 1, 6000 to 7200 UT, the magnetic activity increases to a 3-hourly $K p$ value of 2 . The enhanced activity level pushes the plasmapause earthward; however, it is not until 6600 UT that GEOS 2 crosses the plasmapause. In fact the local time of this crossing is about 2230 LT and based on previous studies corresponds to the satellite crossing an "injection boundary" signature. This boundary is found to have a local time which is related to the level of magnetic activity [Mauk and McIlwain, 1974; Sojka et al., 


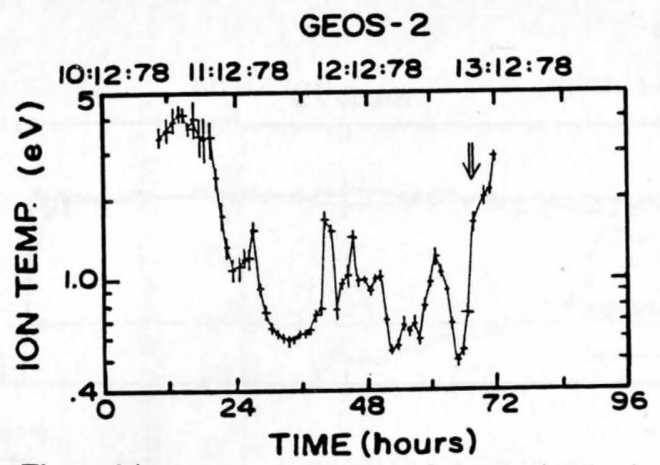

Fig. 3. Thermal ion temperature over a 3-day period beginning at 0000 UT on December 10, 1978. The arrow indicates the end of the quiet period when the plasmapause moves inside the GEOS 2 orbit.

1984]. On crossing this boundary the cold plasma density becomes erratic, ranging from 0.1 to $10 \mathrm{~cm}^{-3}$, while the warm electron density is stable at $1 \mathrm{~cm}^{-3}$ and is isotropic. This latter signature is associated with the plasma sheet.

A second extended quiet period is shown in Figure 2. This 4-day period begins at 0000 UT on March 10, 1980. For this period the local time is given by UT +2 hours, $27 \mathrm{~min}$, which is similar to that for Figure 1. The initial 2-day period in Figure 2 shows fluctuations and trends similar to the earlier period; however, the third and fourth days show remarkably little change in the cold ion density. Over this 44 -hour period the density does not appear to increase significantly, and the fluctuations and anisotropies are relatively mild. This is also reflected in the warm electron densities, which are lower than in the earlier study period, i.e., $\sim 0.01$ to $0.04 \mathrm{~cm}^{-3}$. The average 3-hourly $K p$ on the third and fourth days was either $0+$ or $1+$, which if anything was slightly more disturbed than for the earlier study period. This 2-day period, 4800 to 9200 UT, could indicate that a local region of the plasmasphere is drifting under a corotational electric field and hence is corotating with GEOS 2. Unfortunately, over this period the density remains constant indicating that the plasma flux tube may already be full.

In addition to the cold plasma density the plasmasphere is often characterized by its relatively low temperature. The SPA 15-min survey data yield the cold ion temperature from a calculation of the second moment of the $0-$ to $\sim 10-\mathrm{eV}$ ion distribution function. Figure 3 shows the corresponding cold ion temperature for the first study period shown in Figure 1. The SPA's two analyzers yield temperatures which are equal within experimental uncertainty, and so Figure 3 shows only one temperature. Crosses used to display the temperatures have vertical lengths which correspond to the statistical and analysis uncertainty. The ion temperature in Figure 3 fluctuates from a high of $4 \mathrm{eV}$ to a low of $0.5 \mathrm{eV}$. These temperature fluctuations are in the opposite sense to those found for the cold ion density (see top panel, Figure 1); more significantly, the variations are in phase with the variations in the warm electron density. When the satellite is in the plasma trough, before $2400 \mathrm{UT}$ and again in the plasma sheet beyond $6600 \mathrm{UT}$, the ion temperature is above $1 \mathrm{eV}$. At these times the cold ion density is low, $<1 \mathrm{~cm}^{-3}$, while the warm electron density is between 0.1 and $1 \mathrm{~cm}^{-3}$. When the satellite is deepest in the plasmasphere and the cold ion density is about $100 \mathrm{~cm}^{-3}$, the ion temperature is about 0.5 to $1 \mathrm{eV}$. This latter situation is quite characteristic of the plasmasphere.

Figure 4 shows the cold ion temperatures corresponding to the second study period shown in Figure 2. Again the tempera- ture variations show a marked correlation with the electron density shown in the lower panel of Figure 2. Bet 4800 and 9200 UT the temperature is relatively constant about $0.7 \mathrm{eV}$, with the exception of a brief temperature incre at about 7000 UT. This temperature increase has no cle counterpart in either the cold ion density or warm electro density. Apart from this brief period the 48-hour period is ver characteristic of the plasmasphere with fairly constant densit and cold temperature.

\section{Discussion}

A total of five extended quiet periods were observed b GEOS 2 in its initial 3 years. Two of these periods were shown detail in the previous section. All the periods show the san overall behavior. Initially, the geosynchronous plasma flu tube is highly depleted, with cold plasma densities below 0 . $\mathrm{cm}^{-3}$, the result of an earlier storm. As magnetic condition become quieter, these plasma flux tubes begin to refill, and field-aligned fluxes of ions are observed [Chappell et al., 1982; Sojka et al., 1984]. Once reaching $10 \mathrm{~cm}^{-3}$, the cold ions becom isotropic and continue to refill. After about 2 days the density reaches $\cong 100 \mathrm{~cm}^{-3}$. During this initial refilling when GEOS 2 is just earthward of the plasmapause the associated plasma flux tubes are unlikely to be corotating since a significant ExB convection velocity would have been present. Density variations are as large as an order of magnitude on time scales less than an hour. Such large fluctuations are not new; Chappell [1974] referred to similar but radial density variations as "detached plasma regions." Grebowsky and Chen [1976] modeled such detached regions and spatial plasmapause structures by introducing fluctuating electric fields. Their model results, corresponding to spatial electric field variations, look very similar to those being observed by GEOS 2 . From the corotating in-situ observations it is not possible to uniquely determine if these fluctuations are temporal and/or spatial density irregularities.

Irrespective of the nature of these fluctuations the density in these plasma flux tubes increases from an initial value of less than $1 \mathrm{~cm}^{-3}$ to nearly $100 \mathrm{~cm}^{-3}$ in about 2 days. Figure 5 shows the cold ion density plotted at various LT's as a function of day number from the event reference day. The left panel shows data from Figure 1 starting on December 10, 1978, while the right panel shows data from Figure 2 starting on March 10, 1980. In each case, only one data point is obtained each day for any given LT. For all but two LT's the data in the left panel of Figure 5

\section{GEOS-2}

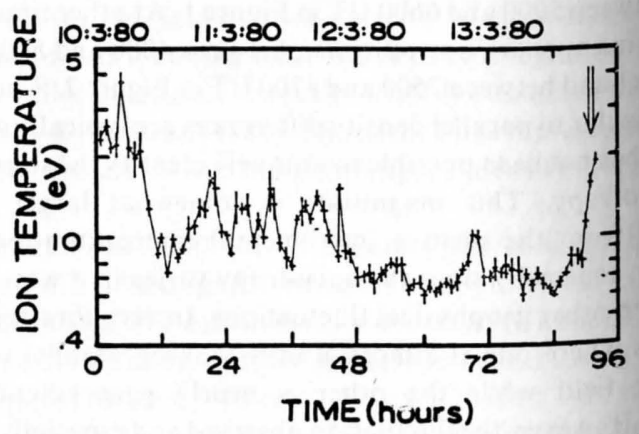

Fig. 4. Thermal ion temperature over a 4-day period beginning al 0000 UT on March 10, 1980. The arrow again indicates the end of th quiet period. 


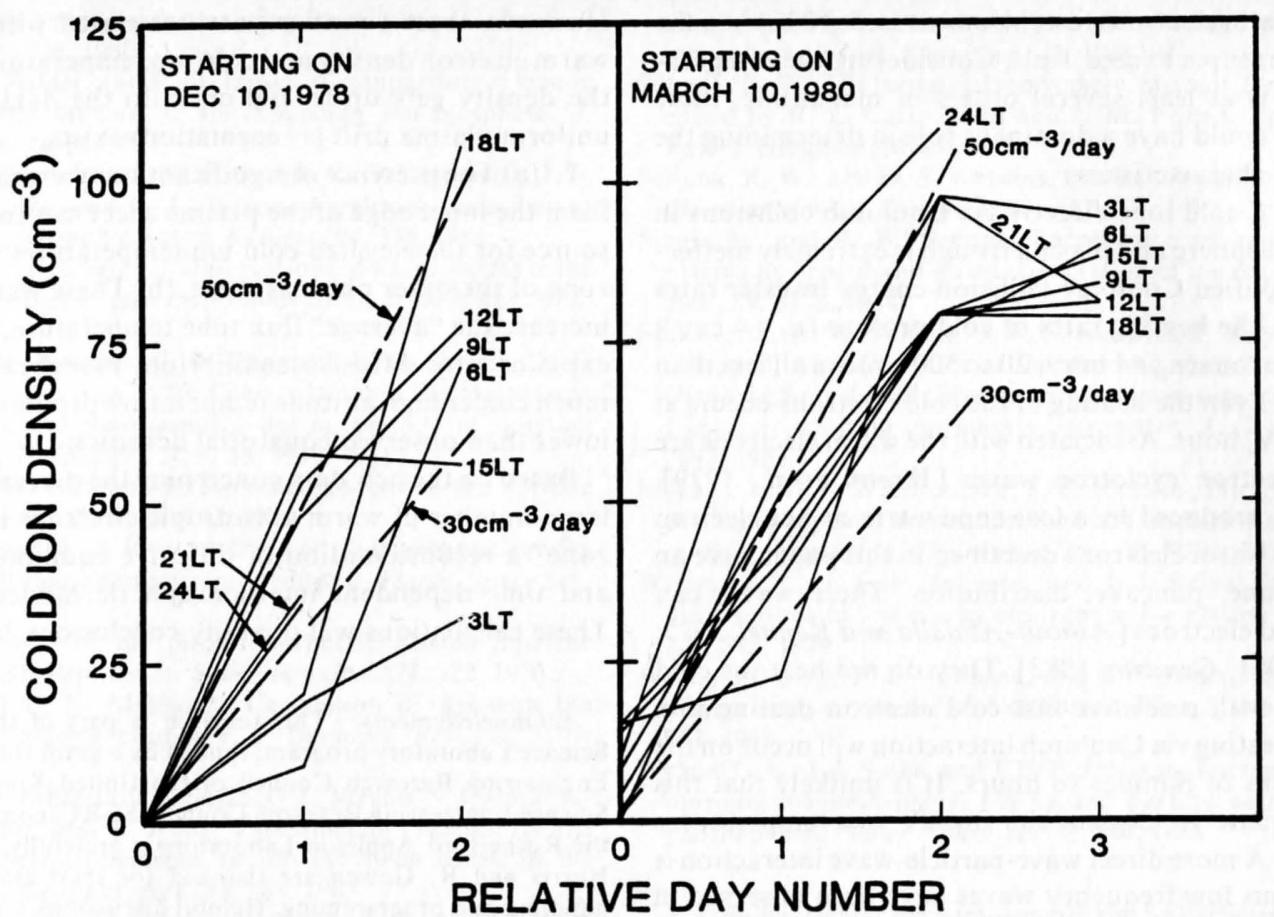

Fig. 5. Cold ion density as a function of day number from December 10, 1978 (left panel), and from March 10, 1980 (right panel). Each panel shows data at 3-hourly LT intervals. Refilling rates of 30 and $50 \mathrm{~cm}^{-3} /$ day are shown as dashed lines in both panels.

have three data points per LT; while the right panel has four except for $2400 \mathrm{LT}$, which has only three data points. The densities are plotted linearly in order to simply display a linear refilling rate if present. Two dashed lines representing 30 and 50 $\mathrm{cm}^{-3} /$ day have been included in both panels. These two levels have been chosen somewhat arbitrarily, but they do span the majority of LT data. This is particularly the case in the right panel for the first three days where all the data except the 2400 LT lie between 30 and $50 \mathrm{~cm}^{-3} /$ day. After the second day the refilling rate changes; in fact, it appears to become zero. The left panel shows significantly greater spread in refilling rates over the 3-day period. Much of this spread is associated with the large amplitude of the fluctuations shown in Figure 1. The data shown in Figure 5, left panel, have been extracted at specific UT's from Figure 1 irrespective of whether that density corresponds to a local fluctuation maximum or minimum. From Figure 5 as well as the earlier Figures 1 and 2 it is evident that two days of data are inadequate to determine with high accuracy a refilling rate; however, these data do show that the average refilling rate does lie between 30 and $50 \mathrm{~cm}^{-3} /$ day for the first two days of refilling. For the right-hand panel the 0600 to $1800 \mathrm{LT}$ data refill at $38 \pm 5 \mathrm{~cm}^{-3} /$ day.

Assuming these refilling rates apply to the equator and that the ions are protons originating lower in the plasmasphere, a simple flux tube mapping to $1000 \mathrm{~km}$ altitude gives fluxes of 2.4 to $3.6 \times 10^{8} \mathrm{~cm}^{-2} \mathrm{~s}^{-1}$. This calculation also assumes both ends of the plasma flux tube supply plasma at the same rate continually for 24 hours. These fluxes are consistent with the proton fluxes conserved by Hoffman and Dodson [1980] for solar maximum conditions in the ionosphere.

Upon reaching $\sim 100 \mathrm{~cm}^{-3}$ the geosynchronous density appears to limit itself. This is shown by the last two days of data in
Fince 2 . However, this limit is statistically difficult to confirm since the remaining GEOS 2 quiet periods are too short in duration to conclusively show this feature. Nevertheless, the data in Figure 2 do show that after the initial 2-day period of refilling to this level the density becomes almost constant and more significantly the density fluctuations become relatively small. Indeed, during this quiet period the other plasma parameters also become less variable, i.e., warm electron density and cold ion temperature. A probable cause of this is that the plasmapause and the associated region of fluctuations are now beyond the geosynchronous location. This would be expected if the plasmasphere is still expanding as it refills. Thus the geosynchronous plasma flux tube is well inside the plasmasphere; indeed, the cold ion temperature of $\sim 0.7 \mathrm{eV}$ would mean that the plasma flux tube is now in the inner cold zone plasmasphere [Bezrukikh and Gringauz, 1976]. In this region the plasma flux tubes are almost corotating, and the convection electric field is negligible. The second 2 days of data shown in Figures 2 and 4 would be consistent with this interpretation.

The initial refilling period is associated with relatively high cold ion temperatures, i.e., from 1 to $5 \mathrm{eV}$. This observation is then consistent with the earlier observations of Bezrukikh and Gringauz [1976], Chappell [1982], and Wrenn et al. [1983]. In fact this region was termed the "hot zone in the outer plasmasphere" by Bezrukikh and Gringauz. In addition to elevated ion temperatures this study shows that this region also contains a significant density of warm electrons in the subkilovolt region (see lower panels, Figures 1 and 2). These warm electrons are associated with or originate from the inner edge of the plasma sheet. As the density of the warm component decreases, so does the cold ion temperature. The existence of this heat source, warm electrons, in the outer zone is then the probable energy source of the elevated cold ion temperatures. Indeed, this heat source naturally arises and is maintained by the drift motions of plasma sheet plasma from the tail earthward. From Figures 1 and 2 in the initial refilling sections 
the density of warm electrons to cold ions exceeds $10 \%$ when the cold ion temperatures exceed $1 \mathrm{eV}$. Considering the temperature difference is at least several orders of magnitude, these warm electrons could have a dominant role in determining the cold ion energy characteristics.

The heating of cold ions directly via Coulomb collisions in the outer plasmasphere and plasma trough is extremely ineffective. Using simplified Coulomb collision energy transfer rates [Schunk, 1983], the heating rates of cold protons $\left(n_{i}=4 \mathrm{~cm}^{-3}\right)$ from warm electrons $\left(n_{e}=1 \mathrm{~cm}^{-3}, 20\right.$ to $\left.500 \mathrm{eV}\right)$ are all less than $0.001 \mathrm{eV} /$ hour. Even the heating of the cold electrons occurs at less than $\sim 0.1 \mathrm{eV} /$ hour. Associated with the warm electrons are electrostatic electron cyclotron waves [Wrenn et al., 1979]. Such waves are produced by a loss cone warm or hot electron population; the warm electrons described in this paper have an extreme loss cone, pancake, distribution. These waves can readily heat cold electrons [Ashour-Abdalla and Kennel, 1975; Horne et al., 1981; Gendrin, 1983]. They do not heat the cold ions; however, with a relative fast cold electron heating rate some cold ion heating via Coulomb interaction will occur on the time scale of tens of minutes to hours. It is unlikely that this would be adequate to explain the few-eV ion temperatures being observed. A more direct wave-particle-wave interaction is required. Various low frequency waves have been observed in the outer equatorialplasmasphere plasma trough region [Ferraut et al., 1978; Norris et al., 1983]. Such waves can also be generated by a pancake type distribution [Ashour-Abdalla and Thorne, 1977], and these waves can directly heat cold ions [Singh and Schunk, 1984]. Since the observed heated cold ions appear to be almost isotropic, the heating must be relatively nonselective in pitch angle since the subsequent cold plasma collisional interaction which would make the distribution isotropic is very slow. Such waves would have to be relatively unpolarized and broadband; otherwise they would lead to ion conic or beam formation [Singh and Schunk, 1984]. To date, no detailed correlation has been searched for between the ULF waves and the heated outer plasmasphere region; hence the latter part of this discussion is speculative.

Another problem associated with the outer plasmasphere is that diffusive equilibrium calculations yield equatorial densities much lower than observed [Chiu et al., 1979; Li et al., 1983]. Under quiet conditions, diffusive equilibrium would be expected to prevail. The probable cause of these lower diffusive equilibrium densities lies with the difficulty in defining the temperature distribution along the plasma flux tube. From the GEOS 2 observations there appears to be a nonnegligible number of warm electrons in the equatorial region. These electrons have pitch angle distributions which confine them near the equator (see Figures 1 and 2, lower panels). By including such electrons, the mean plasma flux tube temperature would increase at high altitudes; the consequence of this for a diffusive equilibrium calculation would be to increase the density at high altitudes.

\section{SUMMARY}

The extended quiet period in-situ observations at the geosynchronous orbit by GEOS 2 gives a number of new insights.

1. (a) The five extended quiet periods, during solar maximum conditions, show the equatorial flux tubes refill at 30 to $50 \mathrm{~cm}^{-3} /$ day. Upon attaining a density of $\sim 100 \mathrm{~cm}^{-3}$, refilling is limited. (b) During the initial refilling (up to $100 \mathrm{~cm}^{-3}$ ) the density variations are huge, up to an order of magnitude.
However, these variations are correlated with changes warm electron density and cold ion temperature. (c) Only the the density gets up to $100 \mathrm{~cm}^{-3}$ do the data indicate that uniform plasma drift ( $\sim$ corotation) exists.

2. (a) The presence of significant numbers of warm ele from the inner edge of the plasma sheet can constitute the hea source for the elevated cold ion temperatures found in the ho zone of the outer plasmasphere. (b) These warm electrons increase the "average" flux tube temperature, which therefore explains why diffusive equilibrium model calculation much colder high altitude temperature profiles get significantly lower than observed equatorial densities.

Based on the new data concerning the presence of a relatively large number of warm anisotropic electrons in the "hot oute zone" a reconsideration of diffusive equilibrium calculations and time dependent interhemispheric models is under way. These calculations will quantify conclusions $2 a$ and $2 b$.

Acknowledgments. This research is part of the Mullard Space Science Laboratory program, funded by a grant from the Science and Engineering Research Council of the United Kingdom. Use of the Science Engineering Research Council (SERC) computing facilities at the Rutherford Appleton Laboratory is gratefully acknowledged. A. Norris and R. Gowen are thanked for their assistance with data handling and programming. Helpful discussions with A. Barakat and $\mathrm{N}$. Singh on the question of cold ion heating are acknowledged.

The Editor thanks Y. T. Chiu and C. R. Chappell for their assistance in evaluating this paper.

\section{REFERENCES}

Ashour-Abdalla, M., and C. F. Kennel, Convective cold upper hybrid instabilities, in Magnetospheric Particles and Fields, edited by B. M. McCormac, pp. 181-196, D. Reidel, Hingham, Mass., 1975.

Ashour-Abdalla, M., and R. M. Thorne, The importance of electrostatic ion-cyclotron instability for quiet-time proton auroral precipitation, Geophys. Res. Lett., 4, 45, 1977.

Banks, P. M., and T. E. Holzer, High-latitude plasma transport: The polar wind, J. Geophys. Res., 74, 6317, 1969.

Bezrukikh, V. V., and K. I. Gringauz, The hot zone in the outer plasmasphere of the Earth, J. Atmos. Terr. Phys., 38, 1085, 1976.

Carpenter, D. L., and C. G. Park, On what ionospheric workers should know about the plasmapause-plasmasphere, Rev. Geophys. Space Phys., 11, 133, 1973.

Chappell, C. R., Recent satellite measurements of the morphology and dynamics of the plasmasphere, Rev. Geophys. Space Phys., 10, 951, 1972.

Chappell, C. R., Detached plasma regions in the magnetosphere, J. Geophys. Res., 79, 1861, 1974.

Chappell, C. R., Initial observations of thermal plasma composition and energetics from Dynamics Explorer-1, Geophys. Res. Lett., 9, 929, 1982.

Chappell, C. R., J. L. Green, J. F. E. Johnson, and J. H. Waite, Jr., Pitch angle variations in magnetospheric thermal plasma-Initial observations from Dynamics Explorer-1, Geophys. Res. Lett., 9, 933, 1982.

Chiu, Y. T., J. G. Luhmann, B. K. Ching, and D. J. Boucher, An equilibrium model of plasmaspheric composition and density, J. Geophys. Res., 84, 909, 1979.

Decreau, P. M. E., J. Etcheto, K. Knott, A. Pedersen, G. L. Wrenn, and D. T. Young, Multi-experiment determination of plasma density and temperature, Space Sci. Rev., 22, 633, 1978.

Frank, L. A., Relationship of the plasma sheet, ring currents, trapping boundary, and plasmapause near the magnetic equator and local midnight, J. Geophys. Res., 76, 2265, 1971.

Gendrin, R., Wave particle interactions as an energy transfer mechanism between different particle species, Space Sci. Rev., 34, 271, 1983.

Grebowsky, J. M., and A. J. Chen, Effects on the plasmasphere of irregular electric fields, Planet. Space Sci., 24, 689, 1976.

Hoffman, J. H., and W. H. Dodson, Light ion concentrations and 
puxes in the polar regions during magnetically quiet times, $J$. Geophys. Res., 85. Fedder, and P. M. Banks, A comparison of kinetic polzer, T. E., J. A. Fetic models of an expanding ion-exosphere, $J$. and hydromas. 76, 2453, 1971.

Geophys. R., P. J. Christiansen, M. P. Gough, K. G. Ronnmark, J. F. Horne, R. B., P. J. Sojka, and G. L. Wrenn, Amplitude variations of E. Johnson, Jotron harmonic waves, Nature, 294, 338, 1981.

electron cycons and . R. Chappell, Observations of warm plasma in the Horwitz, J. Llasma trough at geosynchronous orbit, J. Geophys. Res. dayside plasma 1979.

4. $7075,1979$.

forwitz, J. L., C. R. Baugher, C. R. Chappell, E. G. Shelley, D. T. formal plasma Young, vicinity of the plasmasphere during periods of quieting in the vic activity, J. Geophys. Res., 86, 9989, 1981.

magnetic J., and M. Scherer, Exospheric models of the topside Lemaire, J., Space Sci. Rev., 15, 591, 1974.

W... J. J. Sojka, and W. J. Raitt, A study of plasmaspheric density Lis., 3l, 1315, 1983.

Mrubashi, K., Escape of the polar-ionospheric plasma into the magnetospheric tail, Rep. Ionos. Space Res. Jpn., 24, 322, 1970.

Mauk. B. M., and C. E. Mcllwain, Correlation of $K p$ with the substorm-injected plasma boundary, J. Geophys. Res., 79, 3193, 1974.

Vorris, A. J., J. F. E. Johnson, J. J. Sojka, G. L. Wrenn, N. CornilleauWehrlin, S. Perraut, and A. Roux, Experimental evidence for the acceleration of thermal electrons by ion cyclotron waves in the magnetosphere, J. Geophys. Res., 88, 889, 1983.

Park, C. G., Some features of plasma distribution in the plasmasphere deduced from the antarctic whistlers, J. Geophys. Res., 79, 169, 1974. Perraut, S., R. Gendrin, P. Robert, A. Roux, C. DeVilledary, and D. Jones, ULF waves observed with magnetic and electric sensors on GEOS 1, Space Sci. Rev., 22, 347, 1978.

Raitt, W. J., R. W. Schunk, and P. M. Banks, A comparison of the temperature and density structure in high and low speed thermal proton flows, Planet. Space Sci., 23, 1103, 1975.

Raitt, W. J., R. W. Schunk, and P. M. Banks, The influence of convection electric fields on thermal proton outflow from the ionosphere, Planet. Space Sci., 25, 291, 1977.

Schunk, R. W., The terrestrial ionosphere, in Solar Terrestrial Physics, edited by R. L. Carovillano and J. M. Forbes, pp. 609-676, D. Reidel, Hingham, Mass., 1983.

Schunk, R. W., and D. S. Watkins, Proton temperature anisotropy in the polar wind, J. Geophys. Res., 87, 171, 1982.

Singh, N., and R. W. Schunk, Energization of ions in the auroral plasma by broadband waves: Generation of ion conics, J. Geophys. Res., 89, 5538, 1984.

Sojka, J. J., R. W. Schunk, J. F. E. Johnson, J. H. Waite, and C. R. Chappell, Characteristics of thermal and suprathermal ions associated with the dayside plasma trough as measured by the Dynamics Explorer retarding ion mass spectrometer, J. Geophys. Res., 88, 7895,1983

Sojka, J. J., G. L. Wrenn, and J. F. E. Johnson, Pitch angle properties of magnetospheric thermal protons and satellite sheath interference in their observation, J. Geophys. Res., 89, 9801, 1984.

Wrenn, G. L., J. F. E. Johnson, and J. J. Sojka, Stable "pancake" distributions of low energy electrons in the plasma trough, Nature, 279, 512, 1979.

Wrenn, G. L., J. F. E. Johnson, and J. J. Sojka, The suprathermal plasma analyzers on the ESA GEOS satellites, Space Sci. Instrum., 5, $271,1981$.

Wrenn, G. L., J. J. Sojka, and J. F. E. Johnson, Thermal protons in the morning magnetosphere: Filling and heating near the equatorial plasmapause, Planet. Space Sci. 32, 351, 1983.

J. J. Sojka, Center for Atmospheric and Space Sciences, Utah State University, Logan, UT 84322.

G. L. Wrenn, Mullard Space Science Laboratory, University College London, Holmbury St. Mary, Dorking, Surrey, England.

(Received August 31, 1984;

revised December 10, 1984; accepted January 9, 1985.) 